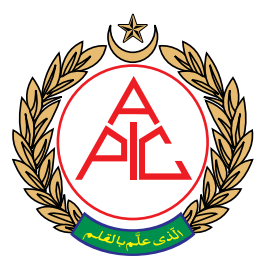

${ }^{1}$ Research Associate; ${ }^{2}$ Assistant Professor Institute of Clinical Psychology, University of Management and Technology, C-II, Johar Town, Lahore, (Pakistan)

Correspondence: $\mathrm{Dr}$ Muhammad Rafiq, Institute of Clinical Psychology, University of Management and Technology,

C-II, Johar Town, Lahore, (Pakistan);

Tel: +92-42-111300200

Ext: 3546 Cell: +92-321-3330471

E-mail: rafiqdar@hotmail.com

Received: 7 September 2018;

Reviewed: 4 October 2018;

Revised: 11 December 2018,

Re-reviewed: 28 December

2018, 31 January 2019;

Accepted: 15 February 2019

\title{
Effect of circle therapy on breast cancer pain management
}

\author{
Saba Maqsood ${ }^{1}$, Muhammad Rafiq, $\mathrm{PhD}^{2}$
}

\begin{abstract}
Objectives \& Background: Recent studies have shown that alternative non pharmacological approaches have significant effect on pain management. Hypnosis has also been seen effective in pain management including cancer pain. The current study was conducted to assess the effect of novel hypnotic approach, called 'circle therapy' on pain due to breast cancer.
\end{abstract}

Methodology: For this study, 60 females suffering from stage 2 breast cancer, undergoing through chemotherapy, were selected by using purposive sampling technique. An intervention plan for breast cancer patients was developed using a hypnosis technique, the 'circle therapy'. Subjective pain rating scale was used for the pre and post assessment of the pain.

Results: Repeated measure ANOVA showed a significant effect of 'circle therapy' on pain caused by breast cancer $(p<0.05)$.

Conclusion: On the basis of this current research, it is concluded that hypnosis has significant effect on pain reduction due to breast cancer. This effect is for short duration; however, this positive effect may be prolonged by increasing the number of hypnotic sessions.

Key words: Cancer pain; Hypnosis; Psychotherapy; Suggestibility; Kappasonian hypnosis; Circle Therapy

Citation: Maqsood S, Rafiq M. Effect of circle therapy on breast cancer pain management. Anaesth. pain \& intensive care 2019; 23(1):63-67

\section{INTRODUCTION}

Pain is the most widely recognized side effect experienced by cancer patients during their course of illness. Pain experienced in cancer patients can be due to the additional side effects of the treatment such as chemotherapy, radiotherapy or surgery. ${ }^{1}$ The sensory intensity of cancer pain vary from patient to patient due to the stage of illness, its duration, location and type of cancer. ${ }^{2}$

The cancer pain has both biological and psychological factors. With respect to numerous cancer patients and survivors, the event of pain may build concern about disease, that it is moving gradually towards a more advanced state. Cancer pain get worse due to interaction of both physical and psychological pain mechanisms as distress is assumed to elevate the pain. ${ }^{3}$

In the course of recent decades, studies have indicated through various examinations that there is a solid relationship between cancer and psychological functioning. The results of these discoveries revealed that cancer patients going through increased pain had relatively high level of anger, anxiety and depression as compared to the patients with less pain. ${ }^{4}$ It was concluded from the results of these researches that the cancer pain is directly associated with more elevated psychological distress, depression, anxiety, fear, and negative mood. ${ }^{5}$

It is frequently accepted that the causes of cancer pain are natural and purely related to biology, therefor it is mostly treated medically. The effect of psychological distress on the physical functioning and quality of life 
of cancer patients is often under rated and therefor health care practitioners don't even try to think about the potential advantages of using psychological interventions for managing cancer related pain. ${ }^{6}$ In this regard, the psychological interventions are considered more helpful in managing the breast cancer pain along with the medical treatment because evidences demonstrated the effectiveness of the psychological interventions in treatment. ${ }^{7}$ Chronic pain is entirely subjective experience and a complex problem. It can persist longer than 3 to 6 months and according to bio-psychosocial model, chronic pain as illness is affected by the biological, psychological and social factors. ${ }^{8}$

Psychological approaches are important in the management of pain, and hypnosis can decrease chronic pain related to medical procedures. There are currently various investigations on hypnosis in management of pain. ${ }^{9}$ Hypnosis has been utilized consistently, both as a medical technique and as a psychotherapeutic intervention. ${ }^{10}$ There are various approaches for induction of state of hypnosis. According to Kappasonian model, hypnosis is a state of subconscious mind created by an overload of message units, disorganizing the critical mind, elicitation of fight/flight reaction leading to hypersuggestible state. ${ }^{11}$

Based on Kappasonian model, a recent developed hypnotic technique called 'circle therapy' has showed significant results in the management of pain of psychological origin ${ }^{12}$. However, no data available on the effect of 'circle therapy' on cancer pain and especially pain due to breast cancer. So, the current research was designed to investigate any effect of 'circle therapy' on reduction of pain due to breast cancer.

\section{METHODOLOGY}

Research design and data collection: The present study was based on experimental research design. Patients with second stage of breast cancer were selected from hospitals in Lahore through purposive sampling technique. Only females with age range of 30-45 years, active treatment, and stage 2 cancer were selected and those with chronic psychological and/or medical illness except breast cancer were excluded.

Procedure: To measure the cancer related pain of the patients before applying the intervention plan, the following parameters was used.

Pain rating scale. The visual subjective pain rating scale was used. It measured the intensity of pain on the scale from 0 to 10 , pre- and posthypnosis.

Suggestibility test. Suggestibility can be defined as the way in which an individual gets the suggestion. Dr. Kappas found that there were significant two kinds of suggestible people i.e. Physical suggestible and emotional suggestible. So, the suggestibility was tested first before any hypnotic suggestion. ${ }^{11}$

In suggestibility test, participants were asked to lift their arm and place the palm in front of face about 10 inches away. They were asked to look at their middle finger and they were suggested with low tone, "Your fingers are separating wider, wider, farther and farther apart from each other". The same suggestion was repeated with other hand but with loud tone. Time needed for separating the fingers was noted for both cases. Participants were emotional suggestible if they were quick responsive with low tone and physical suggestible if they were more responsive to loud tone. ${ }^{11}$

Hypnosis induction: The hypnosis state was induced through a combination of strategies and therapies including progressive relaxation, progressive muscle relaxation and use of relax recliner, and eye fascination was done using Kappasonian model of hypnosis. According to Kappasonian model of hypnosis, an overload of message units (information) is fed to the clients that disorganizes critical thinking process and induces physiological changes (fight/flight reactions) leading to hyper suggestibility (Figure 1). In eye fascination technique, participants were asked to focus on a point on the wall and they were suggested not to blink the eyes and to close the eyes if they feel tired. As they closed their eyes, they were released to put them in hypnotic state. Their hypnosis state further depended through relaxation and imagination. During this state, they were suggested not to speak and give signals only with a finger. While they were following the suggestion, they were asked to do 'circle therapy'.

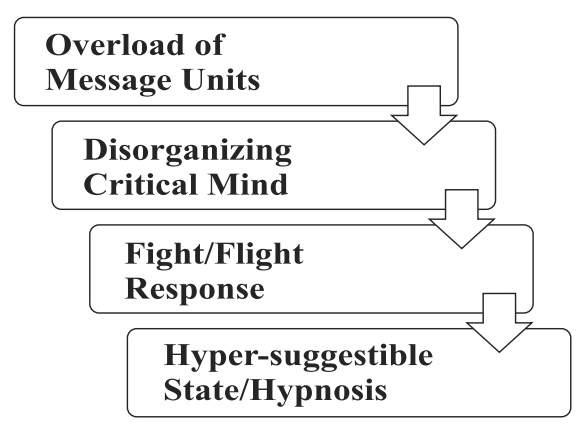

Figure 1: Kappasonian model of hypnosis

ANAESTH, PAIN \& INTENSIVE CARE; VOL 23(1) MARCH 2019 
Application of 'circle therapy': All the participants were provided with a plain paper and a pen. The participants were suggested in the hypnotic state, "You start drawing a circle clockwise as you feel pain is reducing and keep drawing until zero pain". This message was continuously repeated till the pain was reduced or approached zero. In few moments, it was observed by the hypnotist that participants started to draw circle that showed they started to feel lowering pain. They were suggested to stop drawing the circle, as they have no pain. After drawing few circles, they were feeling no or low pain and they were in confident and relaxed state. They were awakened by the hypnotist by counting from 1-5, and at 5 they were suggested to open the eyes. For each participant, a second session was conducted on the next day to see any persistent effect of hypnotic suggestion. Few samples of the circles drawn by the participants during the hvpnotic state are shown in Figure 2.

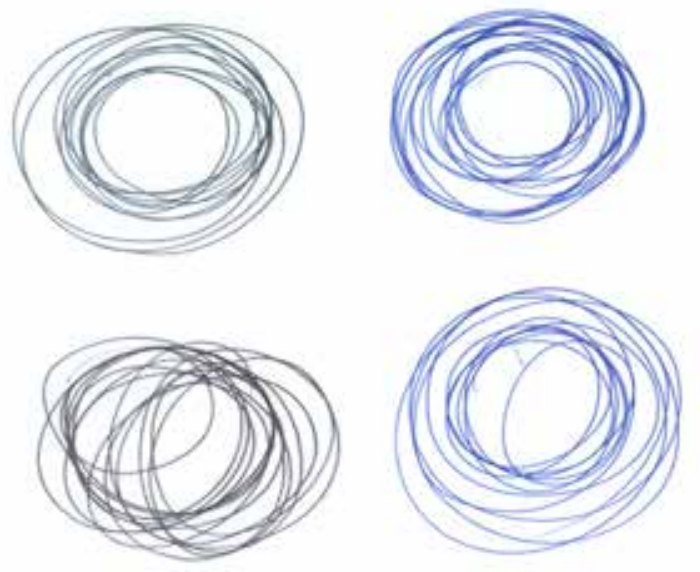

Figure 2: Few samples of the circles drawn by the participants during hypnosis

\section{RESULTS}

Two hypnosis sessions with pre-test and post-test each were done for managing pain and it showed that the pain was decreased at the end of each session.

Table 1: One-way repeated measures ANOVA for the four times of repeated rating across the breast cancer patients $(N=50)$.

\begin{tabular}{|c|c|c|c|c|c|c|c|}
\hline Variable & Measurements & M & SD & L. Bound & U. Bound & $\mathbf{F}$ & p \\
\hline \multirow[t]{2}{*}{ Session One } & Pre rating & 8.38 & .602 & 8.209 & 8.551 & \multirow{4}{*}{1536.62} & \multirow{4}{*}{$0.001^{* \star *}$} \\
\hline & Post rating & 2.62 & .901 & 2.364 & 2.876 & & \\
\hline \multirow{2}{*}{ Session Two } & Pre rating2 & 8.26 & .487 & 8.122 & 8.398 & & \\
\hline & Postrating2 & 1.04 & .727 & .833 & 1.247 & & \\
\hline
\end{tabular}

${ }^{\star \star \star} p<0.001$
Table 1 shows that hypnosis was effective in pain management of patients with cancer, as the four times of repeated rating had significant differences, [Wilk's Lambda $=0.010, \mathrm{~F}=1536.62, p<0.001$, multivariate partial eta squared $=0.99$ ].

Regarding the type of suggestibility, it was indicated that emotional suggestibility is more potent than physical suggestibility, [Wilk's Lambda $=0.59$, partial eta squared $=0.4, p<0.001]$. There therapy was more effective on participants who were taking chemo with supporting medicine than the participants taking supportive medicine alone, [Wilk's Lambda $=0.52$, partial eta squared $=0.48, p<0.001]$.

One-way repeated measures ANOVA showed that there was a significant effect of hypnotic suggestion on breast cancer pain in both the sessions (Table 1).

\section{DISCUSSION}

The present study was focused to see the effect of 'circle therapy', a hypnotic approach on pain due to breast cancer. Recent data on the effect of 'circle therapy' has shown significant reduction in pain but this has not been tried on pain due to cancer. ${ }^{12}$ 'Circle therapy' is based on Kappasonian model of hypnosis that is defined as, hypnosis is induced by overload of message units that blocks the critical mind, the client undergoes in brief state of fight/flight leading to trance that is hyper suggestible state ${ }^{11}$. Here message units are important, which are actually information which disorganize the critical faculty of the participants and putting the clients in subconscious mind which is highly suggestible state of mind.

The use of 'circle therapy' under hypnotic state is useful because when the participant during hypnosis is suggested, "You will start feeling lowering the pain in moments and give a signal with finger when you start lowering the pain and draw a circle clockwise and keep drawing till no pain". As she perceives less pain, she is associated with drawing a circle and with drawing the circle, pain is further reduced till zero or less. As this therapy involves the participants in drawing circles, so is called 'circle therapy'. ${ }^{12}$

Our data showed that 'circle therapy' was more effective on emotional suggestible than physical suggestible. In the past researches, only one way of suggestibility has been explored, however our data for the first time revealed the importance of specific 
type of suggestibility. The concept of suggestibility is explained like, a physical suggestible person is hypersuggestible if physical suggestibility is exercised and vice versa. This model of suggestibility is explained by Kappasonian model of hypnosis and Dr. Kappas stated that there are two major kinds of suggestibility i.e. Physical suggestible and emotional suggestible. ${ }^{11}$

Emotional suggestible are those who prefer suggestion in indirect way or inferred way. However, physical suggestible prefer direct suggestion. As the 'circle therapy' involves the use of inferred suggestions, so that's why 'circle therapy' might be more effective on emotional suggestible than physical suggestible.

Our data indicated that hypnosis was more effective with patients who got chemotherapy with supporting medicine as compared to patients who got supportive medicine alone. A study revealed the significance of hypnosis in managing the chemotherapy induced symptoms of pain, fatigue, insomnia and body aches in an experimental research. Result highlighted a significant decrease in the symptoms of stress, sleep problem and pain in the patients currently going through chemotherapy as compared to the control group who were only given the pain killers. ${ }^{13}$

In terms of age, the result of the current study revealed that the pain of the participants from the age range of 30 to 35 years was reduced considerably more as compared to other two age group. In total, the participants were from three different age ranges 30 to 35 years of age, 35 to 40 years of age and 40 to 55 years of age. The participants from age range 30 to 35 were diagnosed at the early stage, early stage cancer is less intense as compared to the advanced stage. A study from existing literature ${ }^{14}$ revealed that the breast cancer patients with early stages of cancer were able to manage psychological distress, sleep disturbance, fatigue and symptoms of chemo induce pain such as body ache and nausea more significantly as compared to the participants with advanced stages.

The research finding showed no considerable difference of effect of hypnotic suggestion between patients with joint family system or nuclear family systems. Our results also suggested that there is no significant bearing of the level of education of the patient with breast cancer on the effect of hypnosis in reducing pain. Literature suggests that education can be helpful for the patients to cope with the pain, but in our research no significant co-relation was found. This may be due to lack of sufficient data on education variable, as 36 participants of this study were uneducated, 12 had studied up to middle school, one had done matric and only one participant had done intermediate.

\section{CONCLUSION}

On the basis of our study results it is concluded that hypnosis is effective in pain management for patients with breast cancer. It is brief and time limited but also effective for managing secondary pain with zero negative side effects. The differences of the pretest of first session and the pretest of the second sessions (although it is not significant) shows that if more sessions can be conducted, the effect of hypnosis can be prolonged.

Ethical Considerations, ethics were followed in all steps of the research. Written permission was taken from the management and administration of the hospitals. Informed consent was obtained verbally from all the participants and confidentiality was ensured. The participants were subjected to no physical or psychological pressure. Participation in this study was voluntary and all participants had equal right to withdraw from the study at any stage.

\section{Conflict of interest:}

Authors declare no conflict of interest.

\section{Authors' contribution}

SM: Concept, experiments

MR: Concept, manuscript writing 
original article

\section{REFERENCES}

1. Dennert G, Horneber M. Selenium for alleviating the side effects of chemotherapy, radiotherapy and surgery in cancer patients. The Cochrane Library. 2006.

2. Watson $M$, Greer $S$, Rowden L, Gorman C, Robertson B, Bliss JM, et al. Relationships between emotional control, adjustment to cancer and depression and anxiety in breast cancer patients. Psychol Med. 1991;21(1):51-7. [PubMed]

3. Syrjala KL, Jensen MP, Mendoza ME, Yi JC, Fisher HM, Keefe FJ. Psychological a $n \quad d$ behavioral approaches to cancer pain management. J Clin Oncol.

2014;32(16):1703. [PubMed] [Freefull text] doi: 10.1200/JC0.2013.54.4825 [PubMed]

4. Keefe FJ, Abernethy AP, C. Campbell L. Psychological approaches to understanding and treating diseaserelated pain. Annu Rev Psychol. 2005;56:601-30. [PubMed] DOI: 10.1146/annurev. psych.56.091103.070302

5. Portenoy R, Thaler $\mathrm{H}$, Kornblith $\mathrm{A}$, Lepore JM, Friedlander-Klar H, Coyle $\mathrm{N}$, et al. Symptom prevalence, characteristics and distress in a cancer population. Qual Life

Res. 1994;3(3):183-9. [PubMed]

6. Sturgeon M, Wetta-Hall R, Hart T, Good $\mathrm{M}$, Dakhil S. Effects of therapeutic massage on the quality of life among patients with breast cancer during treatment. J Altern Complement Med. 2009 Apr;15(4):37380. [PubMed] doi: 10.1089/ acm.2008.0399.

7. McGregor BA, Antoni MH. Psychological intervention and health outcomes among women treated for breast cancer: a review of stress pathways and biological mediators. Brain Behav Immun. 2009;23(2):159-66. [PubMed] [Free full text] DOI: 10.1016/i.bbi.2008.08.002

8. Kowal J, Wilson KG, Geck CM, Henderson PR, D'Eon JL. Changes in perceived pain severity during interdisciplinary treatment for chronic pain. Pain Res Manag. 2011;16(6):451-6. [PubMed] [Free full text]

9. Elkins G, Jensen MP, Patterson DR. Hypnotherapy for the management of chronic pain. Int J Clin Exp Hypn. 2007;55(3):275-87. [PubMed] [Free full text] DOl:
10. $1080 / 00207140701338621$

10. Barber J, Gitelson J. Cancer pain: Psychological management using hypnosis. CA Cancer J Clin. 1980;30(3):130-6. [PubMed] [Free full text]

11. Kappas JG. Professional hypnotism manual: Introducing physical and emotional suggestibility and sexuality: Panorama Publishing Company; 1987.

12. Rafiq M. Headache management through hypnosis: A case report. Anaesth. pain \& intensive care. 2018;22(2):227-230. [Free full text]

13. Carey MP, Burish TG. Etiology and treatment of the psychological side effects associated with cancer chemotherapy: A critical review and discussion. Psychol bull. 1988;104(3):307-325. [PubMed]

14. Scott JL, Halford WK, Ward BG. United we stand? The effects of a couple-coping intervention on adjustment to early stage breast or gynecological cancer. J Consult Clin Psychol. 2004;72(6):1122. [PubMed] DOI: $10.1037 / 0022-006 \times .72 .6 .1122$ 